\title{
Dual Readout With PWO Crystals and LuAG Crystal Scintillating Fibers
}

\author{
E. Auffray, Member, IEEE, D. Abler, P. Lecoq, Member, IEEE, and G. Mavromanolakis, Member, IEEE
}

\begin{abstract}
One of the main challenges for detectors at future high-energy collider experiments is high-precision measurement of hadrons and jet energy and momentum. Such measurement can be provided by the particle flow approach (PFA) that requires a complex highly segmented calorimeter system to identify and to track all particles in a jet. An alternative so-called dual-readout approach consists of simultaneously recording, in an active medium, scintillation light that is proportional to total energy deposit and Cerenkov light that is proportional to the electromagnetic part only, thus allowing extracting the electromagnetic fraction of the total shower energy on an event-by-event basis. The dual-readout method approach can be implemented using several techniques. The first method proposed uses a copper absorber structure containing two types of fibers: quartz fibers that produce Cerenkov light and plastic scintillating fibers that produce scintillation light. A second method proposed is based on the separation of scintillation and Cerenkov signal produced in homogeneous detector blocks made of scintillating crystals such as bismuth germanate (BGO), lead tungstate (PWO), or others. More recently, a third method has been proposed by our group that uses so-called "meta-crystals" consisting of both Ce-doped and undoped heavy crystal fibers of identical material. The undoped fibers behave as Cerenkov radiator, while Ce-doped fibers behave as scintillators. In this paper, we discuss advantages and inconveniences of all methods and present first results on methods 2 and 3 .
\end{abstract}

Index Terms-Cerenkov, dual readout calorimetry $\mathrm{PbWO}_{4}$ LuAG, inorganic scintiallator.

\section{INTRODUCTION}

$\mathbf{O}$ $\mathrm{NE}$ of the main objectives of future linear $\mathrm{e}^{+} \mathrm{e}^{-}$-colliders is the precise measurement of multijet final states in order to properly identify $\mathrm{W}$ and $\mathrm{Z}$ bosons. The composition of a typical hadronic shower is about $60 \%$ charged hadrons, $30 \%$ photons, and $10 \%$ neutral hadrons. About $70 \%$ of the shower energy is deposited in the hadronic calorimeter. While conventional hadron calorimetry is limited to about $60 \%-70 \% / \sqrt{ } \mathrm{E}$ due to large fluctuations in the fraction of the invisible energy loss through different hadronic processes, the high-precision measurement of hadrons and jets requires a resolution of better than $30 \% / \sqrt{ } \mathrm{E}$.

One possibility to achieve this performance is the particle flow approach. This approach requires tracking calorimeters with extremely high granularity such as silicon tungsten sandwiches aiming at the identification of all particles in a jet

Manuscript received June 30, 2009; revised August 31, 2009 and October 07, 2009; accepted October 23, 2009. Date of current version June 16, 2010.

The authors are with the Department PH, CERN, Geneve 23, Switzerland (e-mail: Etiennette.Auffray@cern.ch; Daniel.Abler@cern.ch; Paul. Lecoq@cern.ch; Georgios.Mavromanolakis@cern.ch)

Color versions of one or more of the figures in this paper are available online at http://ieeexplore.ieee.org.

Digital Object Identifier 10.1109/TNS.2009.2036613 and at the tracking of individual showers [1]. This implies a highly segmented calorimeter with a huge number of channels resulting in complex engineering and integration problems to extract the signals. It also requires sophisticated reconstruction software.

An alternative approach called dual-readout method consists of measuring, independently and over the entire shower volume, both scintillation and Cerenkov light produced in the active medium. While the first one is proportional to the total energy deposited by the shower particles, the latter is produced only by charged, relativistic shower particles that are almost exclusively found in the electromagnetic (em) shower component (dominated by $\pi^{0} \mathrm{~s}$ produced in hadronic showers). Comparing both signals makes it possible to measure the electromagnetic energy fraction, $f_{e m}$ on an event-by-event basis. Several techniques have been proposed for the dual-readout concept.

The first approach proposed by Wigmans et al. (Dream calorimeter [2]) consists of a copper absorber structure containing two types of fibers: quartz fibers that produce Cerenkov light and plastic scintillating fibers that produce scintillation light. However, this approach is limited in terms of energy resolution due to the use of low-density scintillation materials, which requires the insertion of such fibers into a high-Z (e.g., copper) absorbing matrix structure that adds sampling fluctuations to the calorimeter response.

A second method proposed [3] is based on the separation of Cerenkov and scintillation signal produced in homogeneous scintillating crystal block such as bismuth germanate (BGO), lead tungstate (PWO), or others. This approach avoids sampling fluctuations, but there is a nonnegligible interference between the Cerenkov and scintillating signals; a part of Cerenkov emission is reabsorbed by the luminescence center producing scintillation light.

We propose a new and innovative method [4], [5] based on so-called meta-crystals consisting of both Ce-doped and undoped heavy crystal fibers of identical material. The undoped fibers behave as Cerenkov radiator, while Ce-doped fibers behave as scintillators. One can then obtain a very homogeneous, dense, and compact system capable of disentangling the electromagnetic from the hadronic part of a shower in a calorimeter with a uniform radiation length, Moliere radius, and interaction length in the whole volume of the detector.

\section{Dual-Readout Method ApProach Based on PWO BLOCK DETECTORS}

In this method, the separation of Cerenkov and scintillation light produced in homogeneous detector blocks made of heavy 

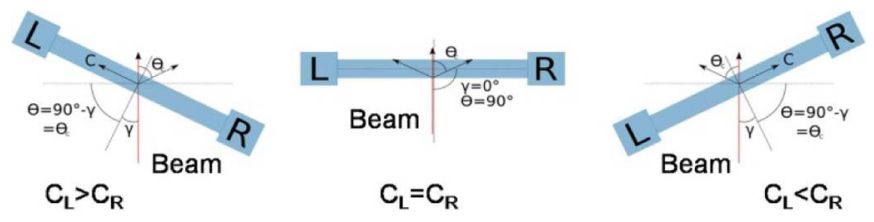

Fig. 1. Principle of determination of the Cerenkov contribution $C_{L}$ and $C_{R}$ are the Cerenkov contribution in the signal measured on PMT left respectively PMT right.

scintillating crystals can be obtained by pulse shape or wavelength discrimination. Indeed the Cerenkov signal is characterized by a prompt signal of few nanoseconds and emission dependency proportional to $\lambda^{-2}$, while the scintillation signal decays slower, with an emission spectra at a well-determined wavelength generally in blue or green region depending on the crystal properties.

Another interesting characteristic of the Cerenkov emission is its nonisotropic emission characteristics, in contrast with the scintillation light. This directional emission can be used to determine the relative fraction of Cerenkov emission in a heavy scintillating crystal. Cerenkov light is produced by charged relativistic particles, and the light is emitted along a cone surface with opening angle $\theta_{\mathrm{c}}$ defined by $\operatorname{Cos} \theta_{\mathrm{c}}=1 /(\beta \mathrm{n})$. The contribution of Cerenkov light relative to the total amount of light varies as a function of the angle between the crystal and the incident particle beam.

\section{A. Methods}

A practical method to determine the Cerenkov contribution is to readout both ends of a crystal by photomultiplier tube (PMT) and to vary the angle between the longitudinal axis of the crystal and the incoming relativistic particle as illustrated in Fig. 1.

The amount of Cerenkov light registered by a PMT mounted at the left and right end-face of the crystal depends on the angle between the longitudinal crystal axis and the incoming relativistic particle due to the direction dependency of the Cerenkov emission. The amount of scintillation light, on the other hand, does not show this dependency since it is emitted in an isotropic manner. A relative difference between the signals in the left (L) and right (R) PMT can therefore be attributed to the presence of Cerenkov light. The Cerenkov contribution to the signal is obtained by measuring the asymmetry $(\mathrm{R}-\mathrm{L}) /(\mathrm{R}+\mathrm{L})$ response as a function of the incident angle.

\section{B. Experimental Setup}

Recently, we performed tests using a $120-\mathrm{GeV}$ electron beam at the $\mathrm{H} 4$ beam line at the CERN SPS using lead tungstate crystals with different doping and thus with different light yield (see Table I). The crystals were mounted in a temperature-controlled copper box in order to protect against light from outside and to stabilize the temperature. The light produced by particles passing through the crystal was read out by two photomultipliers PM2020Q, coupled optically using silicon grease at both ends of the crystal. The copper box was mounted on a platform with a movable support that allowed for rotating the crystal around its geometrical center, as illustrated in Fig. 2. The whole platform was horizontally and vertically adjustable in such a way that the

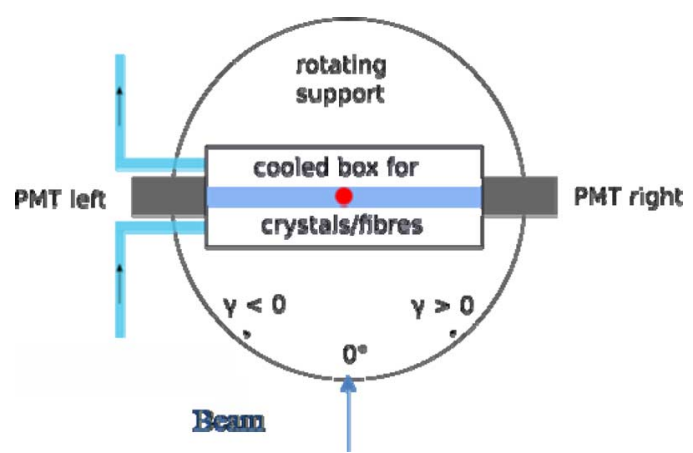

Fig. 2. Experimental setup used in test beam.

geometrical center of the copper box and the crystal could be well aligned with respect to the particle beam.

\section{Results}

Fig. 3 shows the preliminary results of the asymmetry curves obtained for different PWO crystals. The asymmetry is higher for the crystal with lower light yield.

From this asymmetry curve, the fraction of Cerenkov light in each crystal can be calculated.

The signal in each PMT is proportional to the sum of the Cerenkov and scintillation light

$$
\mathrm{PMT}_{\mathrm{R}}=S+C_{\mathrm{R}}, \mathrm{PMT}_{\mathrm{L}}=S+C_{\mathrm{L}}
$$

where $S$ represents the part of the PMT signal originating from the scintillation (identical for both left and right PMT) and $C_{\mathrm{R}}, C_{\mathrm{L}}$ represents the signal originated from Cerenkov light. $C_{\mathrm{R}}$ and $C_{\mathrm{L}}$ vary depending on the incident angle of the particle beam.

The asymmetry (A) of the detector response is defined by

$$
\begin{aligned}
\mathbf{A} & =\frac{\mathrm{PMT}_{\mathrm{R}}-\mathrm{PMT}_{\mathrm{L}}}{\mathrm{PMT}_{\mathrm{R}}+\mathrm{PMT}_{\mathrm{L}}} \\
\mathbf{A} & =\frac{C_{\mathrm{R}}-C_{\mathrm{L}}}{2 \mathbf{S}+C_{\mathrm{R}}+C_{\mathrm{L}}} .
\end{aligned}
$$

When the asymmetry curve reaches its maximum $\left(A_{\max }\right)$ in Fig. 3, there is no Cerenkov signal visible from the left PMT, and the maximum asymmetry $A_{\max }$ is given by

$$
\mathrm{A}_{\max }=\frac{C_{\mathrm{R}}}{2 \mathrm{~S}+C_{\mathrm{R}}}
$$

The Cerenkov fraction of the light $\left(\mathrm{F}_{\mathrm{C}}\right)$ defined as

$$
\mathrm{F}_{\mathrm{C}}=\frac{C_{\mathrm{R}}}{\mathbf{S}+C_{\mathrm{R}}}
$$

Thus, $\mathrm{F}_{\mathrm{C}}$ can be expressed in terms of $\mathrm{A}_{\max }$

$$
\mathrm{F}_{\mathrm{C}}=\frac{2 \mathbf{A}_{\max }}{\mathbf{1}+\mathbf{A}_{\max }} .
$$

The results are presented in Table I. The fraction of Cerenkov varies from $22 \%$ to $13 \%$. This variation of Cerenkov light fraction is in reasonable agreement with the light yield value of each crystal. The lower the scintillation light yield is, the higher the Cerenkov fraction. 


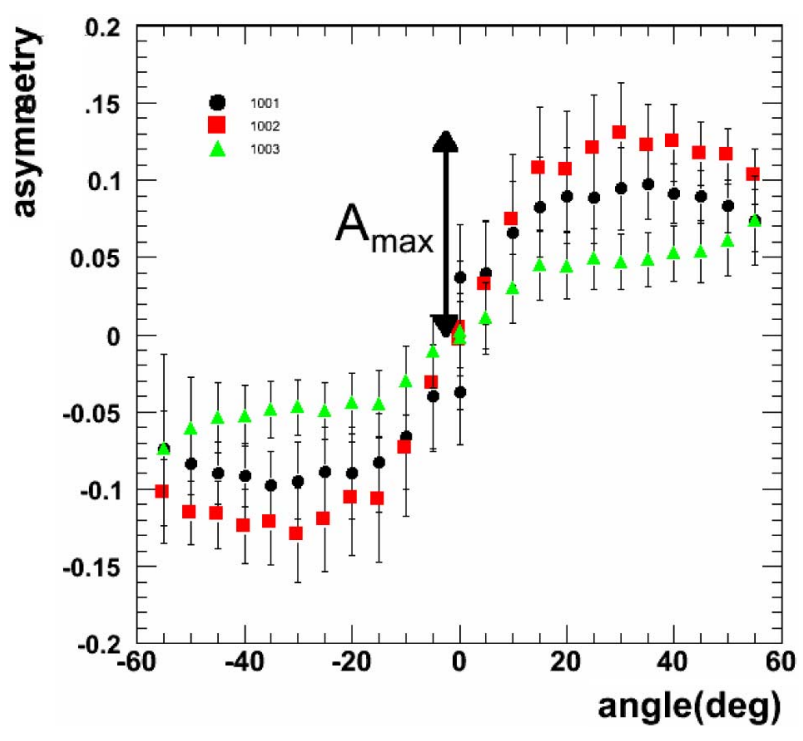

Fig. 3. Asymmetry curves obtained for different types of PWO crystals.

TABLE I

PROPERTIES OF TESTED PWO

\begin{tabular}{lcl}
\hline \hline Crystal number & Light Yield $(\mathrm{pe} / \mathrm{MeV})$ & Cerenkov fraction $(\%)$ \\
\hline PWO1001 & 4.7 & 17.8 \\
PWO1002 & 3.2 & 22.9 \\
PWO1003 & 8.8 & 13.8
\end{tabular}

One of the limitations of this dual-readout approach method is the nonnegligible coupling between Cerenkov and scintillation light. Thus, a part of the Cerenkov light is absorbed by the luminescence centers of the crystal and reemitted as scintillation light. Therefore, we recently proposed a different approach based on the metamaterial concept.

\section{METAMATERIAL DUAL-REAdOUT CONCEPT}

The basic concept of a calorimeter based on heavy scintillating crystal fibers is to subdivide the electromagnetic calorimeter unit volume (typically 1 Moliere radius times 25 radiation lengths) in such a way as to provide more information than the total energy deposit in this volume. The present approach consists of using fibers from a dense, nonintrinsic scintillator with a large band-gap to allow a good UV optical transmission. Undoped fibers of this material will not scintillate, but behave as efficient Cerenkov radiators because of their high refraction index $n$ and their high UV transmission. They will be mainly sensitive to the lightest and therefore fastest charged relativistic particles that will produce Cerenkov light. On the other side, cerium- or praseodymium-doped fibers of the same material will behave as a fast scintillator sensitive to the total energy deposited in the fiber. Combining both types of fibers having the same characteristics results in a very homogeneous, dense, and compact calorimeter system with uniform radiation length, Moliere radius, and interaction length over the entire detector volume capable of disentangling the electromagnetic from the hadronic part in a hadronic shower. Moreover, the fibers can be assembled in metacables in a flexible way, allowing a multitude of different detector geometries.

If the detection of neutrons turns out to substantially improve the overall jet energy resolution, additional scintillation fibers



Fig. 4. Scheme of metacable concept for calorimetry at future linear colliders.

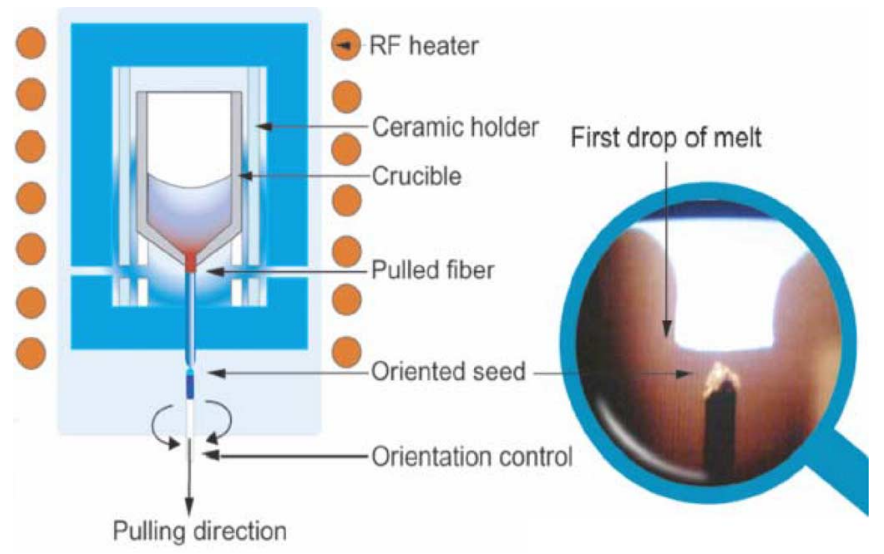

Fig. 5. The micro-pulling down crystal growth technology.

can be inserted made of neutron-sensitive materials such as lithium tetraborate $\mathrm{LBO}\left(\mathrm{Li}_{2} \mathrm{~B}_{4} \mathrm{O}_{6}\right)$, lithium fluoride-based materials like $\mathrm{LiCAF}\left(\mathrm{LiCaAlF}_{6}\right)$, or crystals from the elpasolite family.

A sketch of a metacable concept is presented in Fig. 4.

Highly segmented diffractive optic or microlens plates focus the light emitted by the different fiber types of the metacable on photodetectors such as silicon photomultipliers (SiPMT) or avalanche photodiodes dedicated to each function (total energy, Cerenkov, and neutron components).

\section{Micro-Pulling Down TeChnOlogy}

Micro-pulling down is a crystal growth technique, which allows growing fiber-shaped crystals with a controlled diameter between 0.3 and $3.0 \mathrm{~mm}$ and up to a length of $2 \mathrm{~m}$. Fig. 5 illustrates the crystal fiber production.

At the center bottom of a cylindrical Iridium crucible, a capillary die is present. When the melt drop is formed at the extremity of the capillary, a seed is placed in contact with the drop and the growth process starts. The seed is pulled down continuously with a pulling rate ranging from 0.1 to $0.5 \mathrm{~mm} / \mathrm{min}$ (much faster than Czochralski and Bridgeman method).

By modifying the shape of the capillary die, fibers with different section geometry can be produced, which facilitates the integration of fiber bundles in complex detector systems.

The LPCML Laboratory of the University Claude Bernard in Lyon, France, and Fibercryst Company in Lyon are working on the improvement of this micro-pulling down technology. They have already produced fibers of different sizes of well-known heavy scintillating crystals such as BGO, YAG, LuAG, and LSO [6] with different diameters and lengths. 
TABLE II

LuAG Physico-Chemical Properties

\begin{tabular}{ll}
\hline \hline Structure / Space group & Cubic/ Ia3d \\
Density & $6.73 \mathrm{~g} / \mathrm{cm}^{3}$ \\
Zeff & 62.9 \\
Radiation length Xo & $1.41 \mathrm{~cm}$ \\
Interaction length & $23.3 \mathrm{~cm}$ \\
Hardness & $7.5 \mathrm{Mohs}$ \\
Fracture toughness & $1.1 \mathrm{Mpa} \cdot \mathrm{m}^{1 / 2}$ \\
Cleavage plane & No \\
Melting point & $2260^{\circ} \mathrm{C}$ \\
Thermal expansion & $8.810^{-6} \mathrm{k}^{-1}$ \\
Thermal conductivity & $31 \mathrm{~W} / \mathrm{m}^{\circ} \mathrm{C}$ \\
\hline \hline
\end{tabular}

TABLE III

LUAG OPTICAL PROPERTIES

\begin{tabular}{ll}
\hline \hline $\begin{array}{l}\text { Light Yield for Ce or Pr } \\
\text { doped LuAG }\end{array}$ & $>20000 \mathrm{ph} / \mathrm{MeV}(50 \% \mathrm{NaI})$ \\
Emission wavelength & $535 \mathrm{~nm}$ (Ce doped) \\
& 290,350 (Pr doped) \\
Decay time & $70 \mathrm{~ns}$ (Ce doped) \\
& $20 \mathrm{~ns}$ (Pr doped) \\
Refractive index & $1.842 @ 633 \mathrm{~nm}$ \\
Absorption edge & $177 \mathrm{~nm}$ (undoped) \\
Cerenkov Threshold e energy & $97 \mathrm{KeV}$ \\
Max Cerenkov angle & $57^{\circ}$ \\
Total Reflexion angle & $33^{\circ}$ \\
\hline \hline
\end{tabular}

\section{MATERIAL: LuAG}

Among several known extrinsic scintillators, the lutetium aluminium garnet ( $\mathrm{LuAG}$ ) crystal is considered as a promising candidate for use in the meta-block configuration. Tables II and III summarize the physical, chemical, and optical properties of LuAG.

Figs. 6 and 7 show the transmission curves recently measured at CERN for undoped and cerium-doped LuAG crystals.

With a transmission higher than $60 \%$ from 250 up to $700 \mathrm{~nm}$, the undoped LuAG offers a very good transmission of Cerenkov light without absorption. This large optical transmittance together with its high refraction index $(\mathrm{n}=1.84)$ make LuAG an excellent Cerenkov radiator with an energy threshold of $97 \mathrm{keV}$ to be compared to $190 \mathrm{keV}$ for quartz (Table III).

The transmission of the cerium-doped LuAG crystal shows two strong absorption bands at 350 and $450 \mathrm{~nm}$ corresponding to the excitation wavelength of cerium ion. In the region of cerium emission $(530 \mathrm{~nm})$, the transmission is higher than $80 \%$, resulting in a low attenuation of the scintillation light.

Systematic studies were carried out to assess the influence of cerium concentration on the light yield of LuAG [7]. Fig. 8 shows that with a concentration of $0.36 \%$ of cerium a light yield of about $30000 \mathrm{ph} / \mathrm{MeV}$ is obtained with a decay time of $60 \mathrm{~ns}$ (see Fig. 9). These results show that LuAG-doped cerium is an efficient and fast scintillator.

The optical and scintillation properties, together with a high density of $6.73 \mathrm{~g} / \mathrm{cm}^{3}$, a radiation length of $1.41 \mathrm{~cm}$, and an interaction length of $23.3 \mathrm{~cm}$ that provide a sufficient stopping power necessary for hadronic calorimetry at high energy colliders (Table I), consolidate the potential of LuAG for the metamaterial concept.

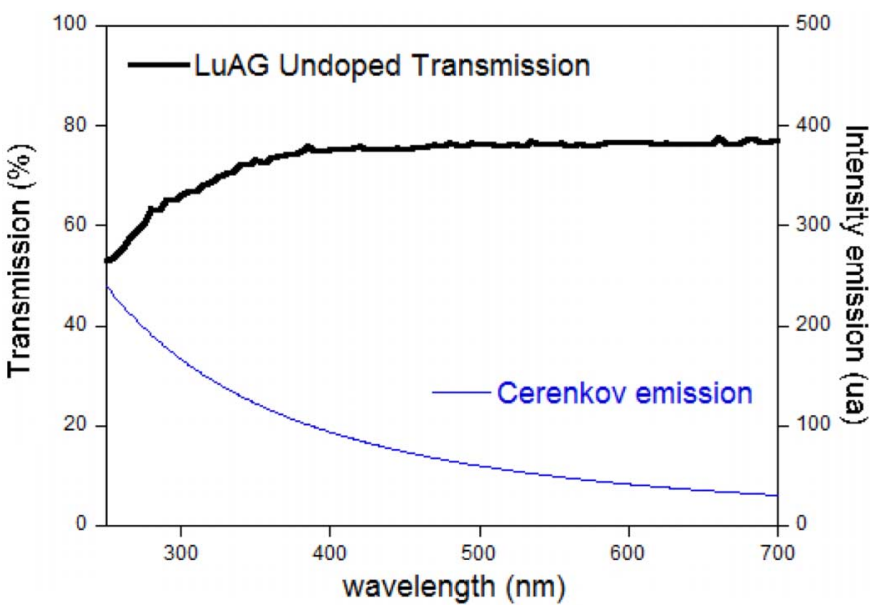

Fig. 6. Transmission of undoped LuAG crystal $\left(2 \times 2 \times 8 \mathrm{~mm}^{3}\right)$. Cerenkov emission wavelength dependence is shown for illustration.

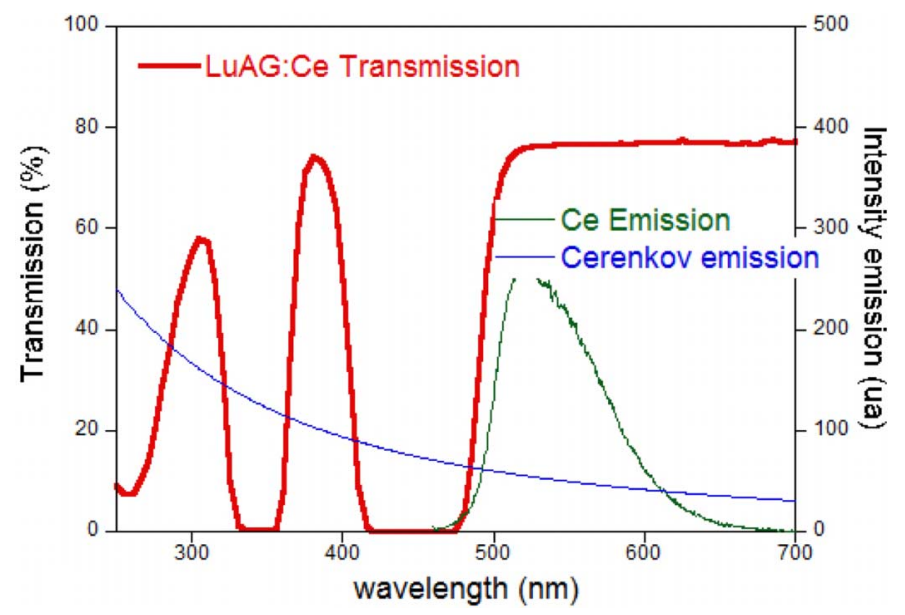

Fig. 7. Transmission and emission spectra of Ce-doped LuAG crystal $\left(2 \times 2 \times 8 \mathrm{~mm}^{3}\right)$. Cerenkov emission wavelength dependence is shown for illustration.

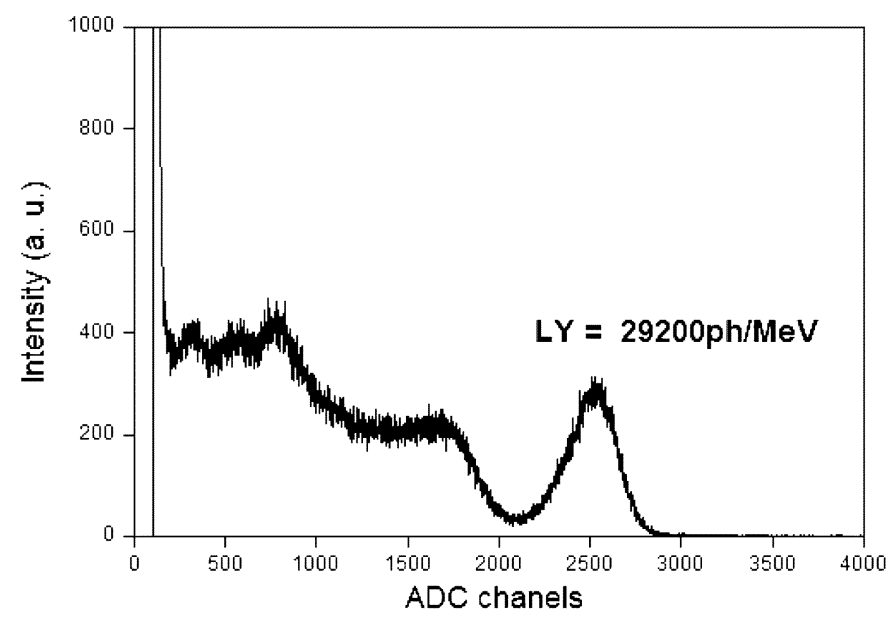

Fig. 8. Light yield spectra of $0.36 \%$ Ce-doped LuAG crystal measured horizontally $\left(2 \times 2 \times 8 \mathrm{~mm}^{3}\right)$.

Moreover, the crystallographic properties of LuAG are very close to yttrium aluminium garnet (YAG), for which the micropulling down grown technique has been extensively used and developed for laser applications. 


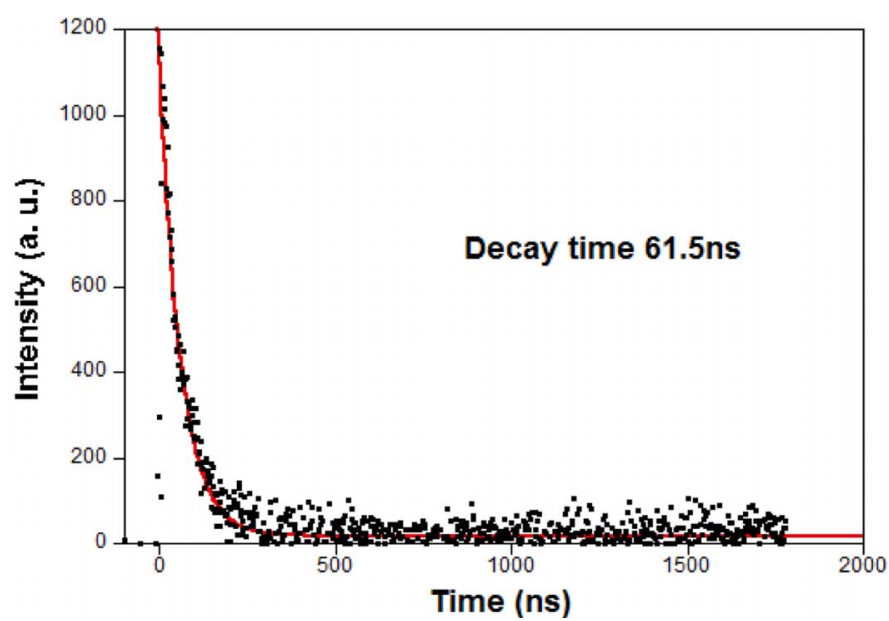

Fig. 9. Decay time spectra of $0.36 \%$ Ce-doped LuAG crystal.

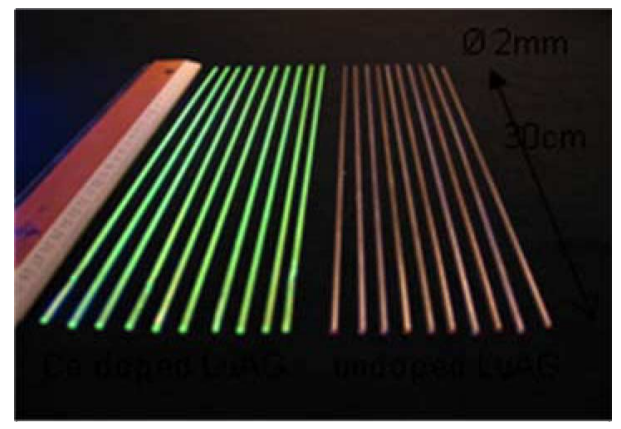

Fig. 10. Lot of Ce-doped and undoped LuAG fibers.

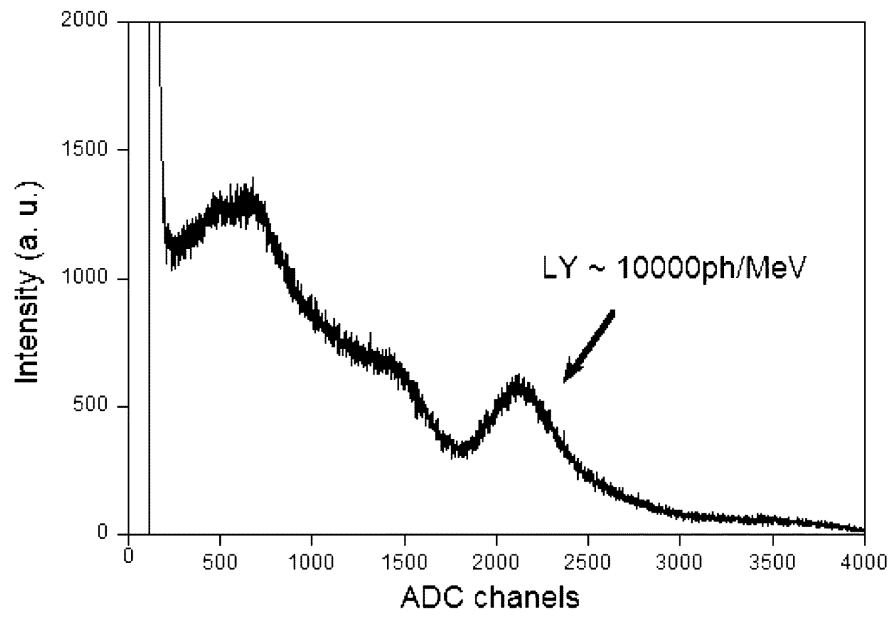

Fig. 11. Light yield spectra of Ce-doped LuAG fiber measured horizontally $\left(\varnothing 2 \times 25 \mathrm{~mm}^{2}\right)$.

\section{FIRST RESULTS}

To assess the suitability of LuAG for a use in a dual-readout metamaterial block for particle calorimetry, a first set of 10 undoped and $10 \mathrm{Ce}$-doped LuAG fibers $(2 \mathrm{~mm}$ diameter and $30 \mathrm{~cm}$ length) was produced by Fibercryst in Lyon, France, and tested at CERN, as shown in Fig. 10.

Fig. 11 shows the light yield spectra measured for a small piece of fiber. A light yield of $10000 \mathrm{ph} / \mathrm{MeV}$ was obtained. The reduction of light compared to the LuAG crystal is mainly

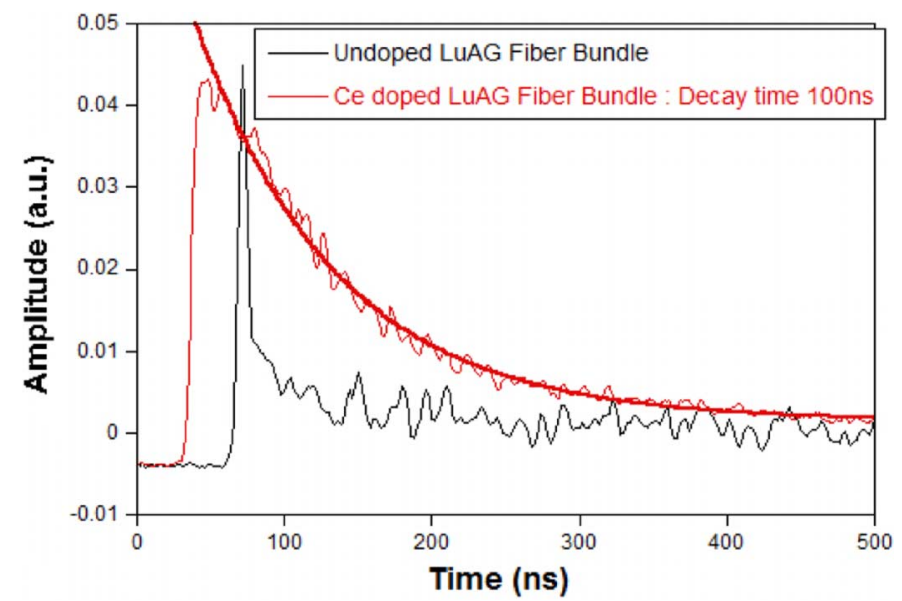

Fig. 12. Pulse spectra obtained with undoped and Ce-doped LuAG fiber bundle with cosmic excitation.

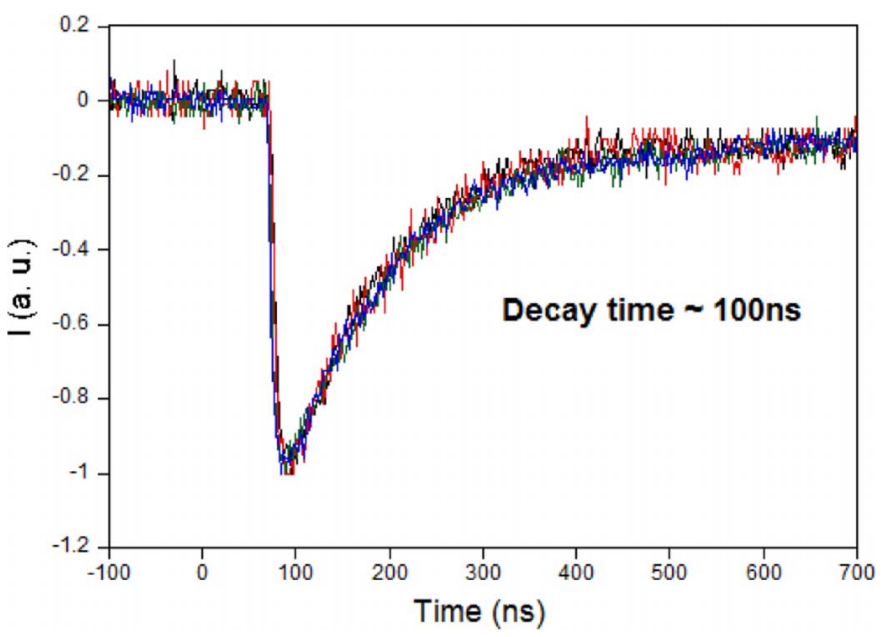

Fig. 13. Pulses obtained with Ce-doped LuAG fiber bundle (scintillation).

due to the lower concentration of cerium $(0.06 \%)$ and a higher attenuation.

Twenty undoped and 20 cerium-doped fibers of $8 \mathrm{~cm}$ length were assembled in bundles supported by a Teflon structure. Both bundles were exposed to cosmic rays. Fig. 12 shows the measured pulse shape spectra of Cerenkov and scintillation fiber bundle. As expected, while the undoped fiber shows a rapidly decaying Cerenkov signal, one clearly recognizes the scintillating signal for the Ce-doped fiber with a decay time of about $100 \mathrm{~ns}$. The slower decay time observed in the fibers compared to the one obtained with crystal sample is probably due to presence of some defects.

The same bundles have been then exposed to a $120-\mathrm{GeV}$ electron beam in the $\mathrm{H} 4$ beam line of the CERN SPS. We used the same setup used for the PWO crystals as described in Section II. Two PMT 2020Q were coupled at both ends of the bundle, which was inserted in the copper box.

Figs. 13 and 14 show pulses extracted for the Ce-doped LuAG bundle and undoped LuAG bundle, respectively, at a temperature of $20^{\circ} \mathrm{C}$. Both fiber types show a clear response to the electron beam. The slow component observed for undoped LuAG fiber on Fig. 14 is due to the self-trapped exciton emission. 


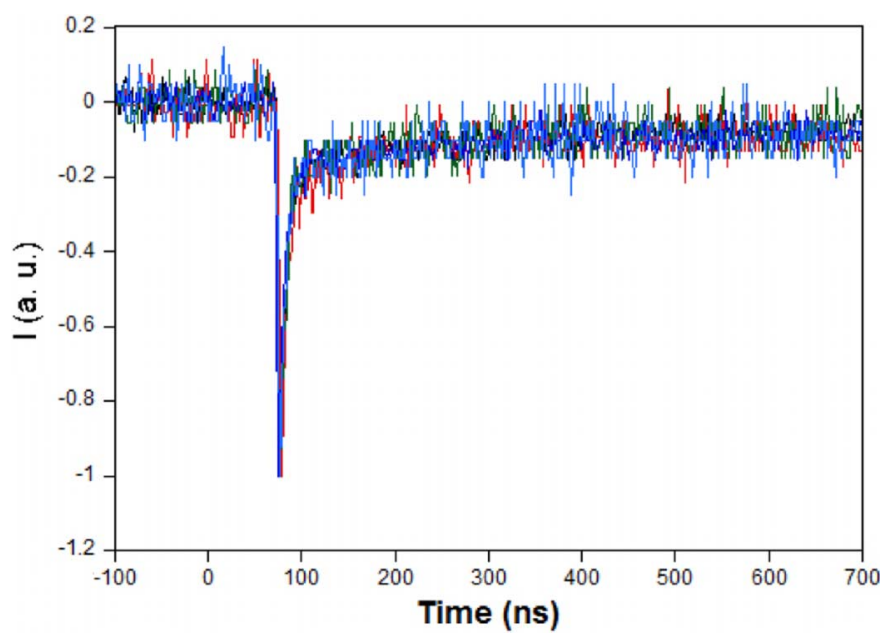

Fig. 14. Pulses obtained with undoped LuAG fiber bundle (Cerenkov).

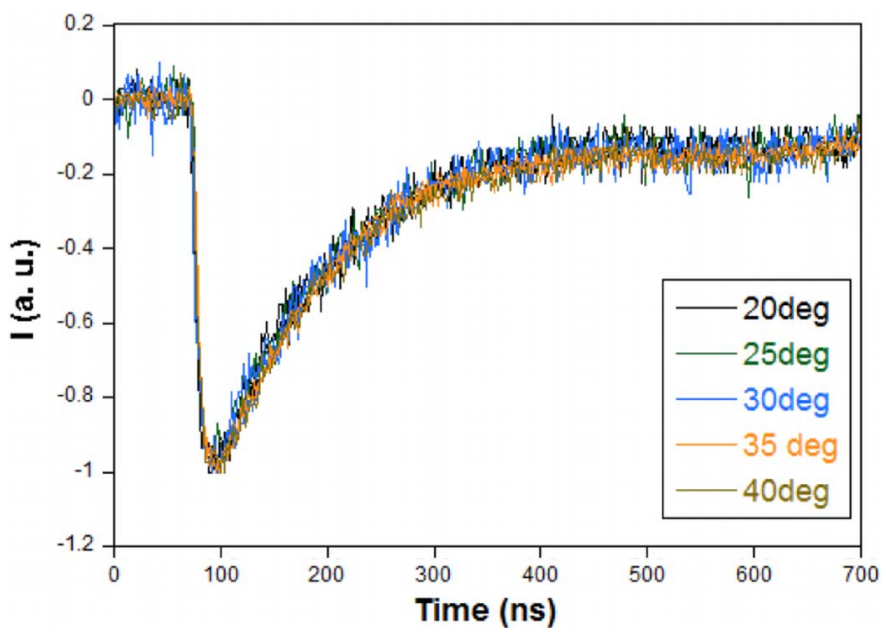

Fig. 15. Pulses obtained at different temperatures for Ce-doped LuAG fiber bundle (scintillation).

The temperature dependence of the decay time of the scintillating signal is shown in Fig. 15 for temperatures between $20^{\circ}$ and $40^{\circ}$. No significant difference can be seen between the scintillation decay time constants in this temperature range.

\section{CONCLUSION AND OUTLOOK}

Recent developments in crystal production technologies allow for producing long crystal fibers of different diameters and section shape. The use of crystal fibers offers new perspectives for the design of particle detectors suitable for calorimetry at future linear colliders. The combination of Ce-doped with undoped LuAG fibers in a bundle allows a fine segmentation of the calorimeter and the extraction of the different components of hadronic showers (electromagnetic, charged, and neutral hadrons) on an event-by-event basis.

A first attempt to assess the performance of such fibers has been carried out in a test beam at CERN; the expected scintillation and Cerenkov signals were observed. Detailed analysis of the test beam data is currently ongoing. In parallel, simulation work will be carried out in order to optimize the detector geometry and granularity for a precise determination of the electromagnetic fraction of the energy deposition.

\section{ACKNOWLEDGMENT}

The authors would like to thank the technical staff from the CERN PH-CMX group, as well as S. Brunner, G. Chevenier, J. Daguin, B. Frisch, and A. Knapitsch, who helped them with the preparation of the test beam and the data taking. They would like also to thank the company Fibercryst, who produced the fibers; A. Petrosyan (from Institute for Physical Research, Astharak, Armenia), who provided the LuAG crystal samples; and M. Korzhik from INP Minsk and the Bogorodistk Technical-Chemical plant. who produced PWO samples. This work is carried out in the frame of the Crystal Clear Collaboartion at CERN.

\section{REFERENCES}

[1] "The CALICE collaboration, design and electronics commissioning of the physics prototype of a $\mathrm{Si}-\mathrm{W}$ electromagnetic calorimeter for the international linear collider," 2008_JINST_3_P08001, 2008.

[2] N. Akchurin, O. Atramentov, K. Carrell, K. Z. Gümüs, J. Hauptman, H. Kim, H. P. Paar, A. Penzo, and R. Wigmans, "Separation of scintillation and Cherenkov light in an optical calorimeter," Nucl. Instrum. Methods Phys. Res. A, vol. 550, pp. 185-200, 2005.

[3] R. Wigmans, "Recent results from the DREAM project," in Proc. Calor08 Conf., J. Phys., Conf. Ser., 2009, vol. 160, p. 012018.

[4] P. Lecoq, "New crystal technologies for novel calorimeter concepts," in Proc. Calor08 Conf., J. Phys., Conf. Ser., 2009, vol. 160, p. 012016.

[5] P. Lecoq, "Metamaterials for novel X- or $\gamma$-ray detector designs," in Proc. NSSS_MIC IEEE Conf., Dresden, Germany, 2008, pp. 1405-1409.

[6] P. Anfre;acute;, C. Dujardin, J. M. Fourmigué, B. Hautefeuille, K. Lebbou, C. Pedrini, D. Perrodin, and O. Tillement, "Evaluation of fiber-shaped LYSO for double readout gamma photon detection," IEEE Trans. Nucl. Sci., vol. 54, no. 2, pp. 391-397, Apr. 2007.

[7] D. Abler and C. Dujardin et al., "LuAG:CE fibers for high energy calorimetry," presented at the SCINT 2009 Conf., Jiju, Korea, Jun. 2009 , submitted for publication. 